\title{
Impatience and Time Inconsistency in Discounting Models
}

\author{
Haewon Yoon \\ Kelley School of Business, Indiana University, Indianapolis, Indiana 46204, haeyoon@iu.edu
}

Extant theories of intertemporal choice entangle two aspects of time preference: impatience and time inconsistency. Impatient people focus on present consumption without worrying too much about the future; they may spend freely and avoid exercise. An outsider might question their choices, but impatient people do not experience conflict over those choices. By contrast, people who are time-inconsistent intend to save and exercise, but they fail to do so when temptation is proximate. Such individuals are conflicted; their preferences today differ from their preferences tomorrow. I characterize the interaction between impatience and time inconsistency in three leading models of temporal discounting that go beyond the exponential model, which does not predict time inconsistency at any level of impatience. The quasi-hyperbolic model predicts that time inconsistency increases with patience, while the hyperbolic model makes the opposite prediction. The constant-sensitivity model predicts that time inconsistency peaks at a moderate level of impatience. The results of an experiment using real monetary consequences with delays of up to one year align most closely with the prediction of the constant-sensitivity model.

Keywords: intertemporal choice; hyperbolic discounting; impatience; time inconsistency

\section{Introduction}

The phenomenon of time inconsistency in intertemporal choice is one of the most influential findings in the judgment and decision-making literature (Ainslie 1975; DellaVigna and Malmendier 2006; Hoch and Loewenstein 1991; O'Donoghue and Rabin 1999; Thaler 1981; Tversky et al. 1990). Over the last three decades, several different behavioral models have been proposed to account for time inconsistency in decision making. In this paper, I consider a baseline exponential model, which considers impatience but does not accommodate time inconsistency, and three prominent discounting modelshyperbolic (Mazur 1987), quasi-hyperbolic (Laibson 1997), and constant-sensitivity (Ebert and Prelec 2007) — that consider both impatience and time inconsistency. I compare each model's theoretical predictions with empirical data.

This is the author's manuscript of the article published in final edited form as:

Yoon, H. (2020). Impatience and Time Inconsistency in Discounting Models. Management Science, 66(12), 5485-6064. https://doi.org/10.1287/mnsc.2019.3496 
The phenomenon of time inconsistency occurs when someone makes contradictory choices at two different points in time. In the current study, I categorize time inconsistency as an initial preference for a larger, later option followed by a preference for a smaller, sooner option - a reversal of the initial preference. On the surface, this is a manifestation of our dynamically-inconsistent preferences over time - we are relatively patient when the consequences are far from now, but impatient when the consequences are imminent. Anecdotally, we experience these inconsistencies in our daily lives; for instance, we might sign up for a gym membership but then not attend regularly (DellaVigna and Malmendier 2006).

However, not everyone reverses their initial decisions in this way. Generally, patient people are unlikely to demonstrate time inconsistency because they still find the larger, later option attractive even when the smaller option is immediately available (e.g., some people actually go to the gym early in the morning, every day, rather than snoozing the alarm clock and sleeping in). Similarly, impatient people are unlikely to demonstrate time inconsistency because they never consider choosing the later outcome (e.g., they never sign up for the gym in the first place). Impatience can play a significant role in the phenomenon of time inconsistency. The current study explores how the level of impatience is related to time inconsistency and how popular discounting models predict this interaction. As I next detail, the disparate ways in which the behavioral models operationalize impatience and time inconsistency yield important differences.

The hyperbolic model specifies the weighting of a future outcome at time $t$ via the function $f(t)=$ $1 /(1+k t)$, where $k$ represents the decision maker's impatience. When $k=0$ (i.e., not impatient), the function reduces to 1 , meaning that the decision maker weighs all periods equally and thus will prefer the larger, later option. As $k$ rises (i.e., impatience increases), the later outcome receives less weight than the sooner outcome, indicating a preference for the smaller, sooner option over the larger, later option. At the same time, $k$ increases the convexity of the curve by increasing the denominator of the function, creating time inconsistency.

The quasi-hyperbolic model specifies the weighting of a future outcome at time $t$ via the function $f(t)=\beta \delta^{t}$, where $\delta$ is the discount factor, which characterizes impatience (smaller $\delta$ indicates a preference for the smaller, sooner option over the larger, later option), and $\beta$ is the present-bias parameter, which incorporates time inconsistency by assigning less weight to the later outcome (i.e., $t>0$ ) but not to the immediate outcome (i.e., $t=0)$. Thus, smaller $\beta(0<\beta<1)$ leads to more time inconsistency. The model does not demonstrate time inconsistency when $\beta=1$. 
Finally, the constant-sensitivity model specifies the weighting of a future outcome at time $t$ via the function $f(t)=\exp \left(-(a t)^{b}\right)$, where $a$ is the discount rate, which characterizes impatience (higher $a$ indicates a preference for the smaller, sooner option over the larger, later option), and $b$ is the time sensitivity parameter, which incorporates time inconsistency by adjusting the sensitivity of perceived time. When $b$ is 1 , meaning that perceived time is the same as objective time, the model does not demonstrate time inconsistency. When $b<1$, meaning that perceived time is insensitive to objective time, the model starts to create time inconsistency, with smaller $b$ demonstrating more time inconsistency. ${ }^{1}$

Impatience and time inconsistency are qualitatively different concepts, but the hyperbolic model demonstrates impatience and time inconsistency using one parameter. The quasi-hyperbolic and constant sensitivity models use two separate parameters to describe impatience and time inconsistency, and the role of each parameter is straightforward when isolated from other factors. It is not immediately obvious, however, how the two parameters interact with each other during an evaluation of two future outcomes that involve other variables - such as the amount and the delay of these two options - that also influence time inconsistency. The current study considers various factors that influence time inconsistency, and it demonstrates each model's prediction of time inconsistency with varying levels of impatience. I find evidence consistent with the constant-sensitivity model's prediction.

\section{Analysis Framework}

In this section, I propose a model analysis framework to examine the time-consistent and timeinconsistent choice patterns predicted by each discounting model. This framework is in line with Prelec (2004), who approached this issue using the Pratt-Arrow convexity of the log of the discounting function. A major difference is that I use the cardinality of the discounting function and a simulation approach to reveal differences in the models' predictions of choice patterns, which I will describe in the next section.

The behavioral models account for both time-inconsistent preferences, which I hereafter refer to as the L-S pattern (i.e., preferring the larger, later option first, but then switching to the smaller, sooner option), and time-consistent preferences, which may fit one of two patterns: the L-L pattern represents a time-consistent preference for the larger, later option, while the S-S pattern represents a time-consistent preference for the smaller, sooner option. My study examines the proportions of these three choice

\footnotetext{
${ }^{1}$ Note that Herrnstein (1990) originally hinted at time sensitivity as the psychological principle behind timeinconsistent preferences. Herrnstein identified the time-perception gap as a possible psychological mechanism for the time-inconsistency phenomenon - specifically, "a systematic psychological distortion of time perception" (p. 359). That is, the perceived or projected duration of time can differ from the actual, objective duration, and this discrepancy can affect choices between delayed outcomes (Zauberman et al. 2009).
} 
patterns (L-L, L-S, and S-S) across varying intertemporal choice questions and investigates how the proportions vary with the level of impatience. ${ }^{2}$

This analysis framework creates a systematic list of possible combinations of intertemporal choice questions and evaluates them using the aforementioned discounting models. The analysis also varies specific parameters in each discounting model: $r$ in the exponential model, ${ }^{3} k$ in the hyperbolic model, $\beta$ and $\delta$ in the quasi-hyperbolic model, and $a$ and $b$ in the constant-sensitivity model. Thus, the analysis framework demonstrates how each discounting model predicts three choice patterns-L-L and S$\mathrm{S}$, both time-consistent, and L-S, time-inconsistent-across different intertemporal choices, and it demonstrates how the prevalence of each choice pattern varies with the aforementioned parameters.

\subsection{Three Steps in the Analysis Framework}

\section{Step 1. Creating intertemporal choice questions}

To elicit L-L, L-S, and S-S patterns, I created a series of intertemporal choice questions that varied the delay for the smaller, sooner option $\left(S_{\text {delay }}\right)$ while holding constant the amount of the larger, later option $(L)$, the amount of the smaller, sooner option $(S)$, and the difference in the delays of the two options ( $\Delta=L_{\text {delay }}-S_{\text {delay }}$; see the table in Figure 1). In this series of intertemporal choice questions, the same choice outcome was presented iteratively over time until $S_{\text {delay }}$ became zero. Note that the calendar date of the two options did not change, but the delays became shorter as time elapsed. For instance, imagine two options: $\$ 300,60$ days from now, or $\$ 500,100$ days from now ( $T_{1}$ and the top row of the table in Figure 1). After many iterations, the choice eventually becomes $\$ 300$, today or $\$ 500,40$ days from now ( $\mathrm{T}_{2}$ and the bottom row of the table in Figure 1). In this example, $L(\$ 500), S(\$ 300)$, and $\Delta(40$ days) are held constant, whereas $S_{\text {delay }}$ shifts from 60 days to 0 days (i.e., today).

This series of intertemporal choice questions can reveal one of the three choice patterns. If a decision maker prefers the larger, later option when $S_{\text {delay }}$ is longer (e.g., preferring $\$ 500$ in 100 days over $\$ 300$ in 60 days), but prefers the smaller, sooner option when $S_{\text {delay }}$ becomes shorter (e.g., preferring $\$ 300$ today over $\$ 500$ in 40 days), then she demonstrates the L-S pattern. On the other hand, if she prefers the smaller, sooner option for every choice in the series, then she demonstrates the S-S pattern; if she prefers the larger, later option for every choice in the series, then she demonstrates the L-L pattern.

\footnotetext{
${ }^{2}$ In addition, the S-L pattern indicates a preference reversal from a smaller, sooner option to a larger, later option (also known as reverse time inconsistency). This may occur when people demonstrate increasing impatience (Sayman and Oncüler 2009) but is improbable in hyperbolic models that demonstrate decreasing impatience (see the discussion of reverse preference reversal in Appendix A).

${ }^{3}$ The exponential model (Samuelson 1937) specifies the weighting of a future outcome at time $t$ via the function $f(t)$ $=\exp (-(r t))$ where $r$ is the discount rate, which represents impatience (higher $r$ indicates a preference for the smaller, sooner option over the larger, later option). The exponential model does not demonstrate time inconsistency.
} 
I then expanded the series of intertemporal choice questions by varying $L, S$, and $\Delta$. For the sake of simplicity, I systematically varied $\Delta$ and $L / S$ (the ratio of the amount of the larger, later option to the amount of the smaller, sooner option) instead of varying all three variables independently, because the discounting models in the current study are scale-invariant. This yielded the three-dimensional parameter space as illustrated in Figure 1. In this parameter space, a specific coordinate represents a single intertemporal choice question. For example, a combination of $L / S=\$ 500 / \$ 300, \Delta=40$ days, and $S_{\text {delay }}=$ 60 days ( $T_{1}$ in Figure 1) represents a choice between $\$ 300$ in 60 days and $\$ 500$ in 100 days. Within this three-dimensional parameter space, the current analysis framework created a list of all possible combinations of intertemporal choice questions for scale-invariant discounting models.

\section{Step 2. Evaluating intertemporal choice questions}

Next, I used each discounting model to evaluate each intertemporal choice question in turn. For each discounting model, I expanded the range of the model's parameter(s) until the model reached an asymptote on either the low end (for the L-L pattern) or the high end (for the S-S pattern), meaning that further changes in the parameter(s) were no longer meaningful. This procedure yielded the following parameter ranges: $r=10^{-6} \sim 10^{1}$ in the exponential model; $k=10^{-5} \sim 10^{2}$ in the hyperbolic model; $\beta=0 \sim$ 1 and $\delta=10^{-4} \sim 10^{0}$ in the quasi-hyperbolic model; and $a=10^{-15} \sim 10^{10}$ and $b=0 \sim 1$ in the constantsensitivity model. I used 100,000 log-spaced intervals within the ranges of $r, k, \delta$, and $a$ (e.g., $r_{1}=10^{-6}, r_{2}$ $\left.=10^{-5.9999}, \ldots, r_{100,000}=10^{1}\right)$ and 11 linearly-spaced intervals within the ranges of $\beta$ and $b$ (e.g., $\beta_{1}=0, \beta_{2}=$ $\left.0.1, \ldots, \beta_{11}=1\right)$.

\section{Step 3. Categorizing responses into choice patterns}

Last, I categorized each response for each $L / S$ and $\Delta$ combination as one of the three choice patterns (S-S, L-L, or L-S). As depicted in Figure 1, each $L / S$ and $\Delta$ combination yielded a series of intertemporal choice questions (represented as vertically stacked boxes from $T_{1}$ to $T_{2}$ ). The responses to these choice questions were categorized as one of the three choice patterns (S-S, L-L, or L-S), and this categorization procedure was repeated for all $L / S$ and $\Delta$ combinations in the parameter space. Thus, as illustrated in Figure 2, this process yielded a two-dimensional "slice" defined by the $\Delta$ and $L / S$ parameters, color-coded for the three choice patterns (red: S-S pattern, blue: L-L pattern, and green: L-S pattern), and with one slice for each model parameter value. This categorization process was repeated for each model with different parameter ranges, creating many two-dimensional "slices" as in Figure 2.

Finally, I simplified the relationship between the parameter(s) of each model and the choice patterns by collapsing the $L / S$ and $\Delta$ dimensions in Figure 2, and then by revealing the proportion of each choice pattern with varying model parameter values in Figure 3. Figure 3 represents the final results of the current analysis framework. 


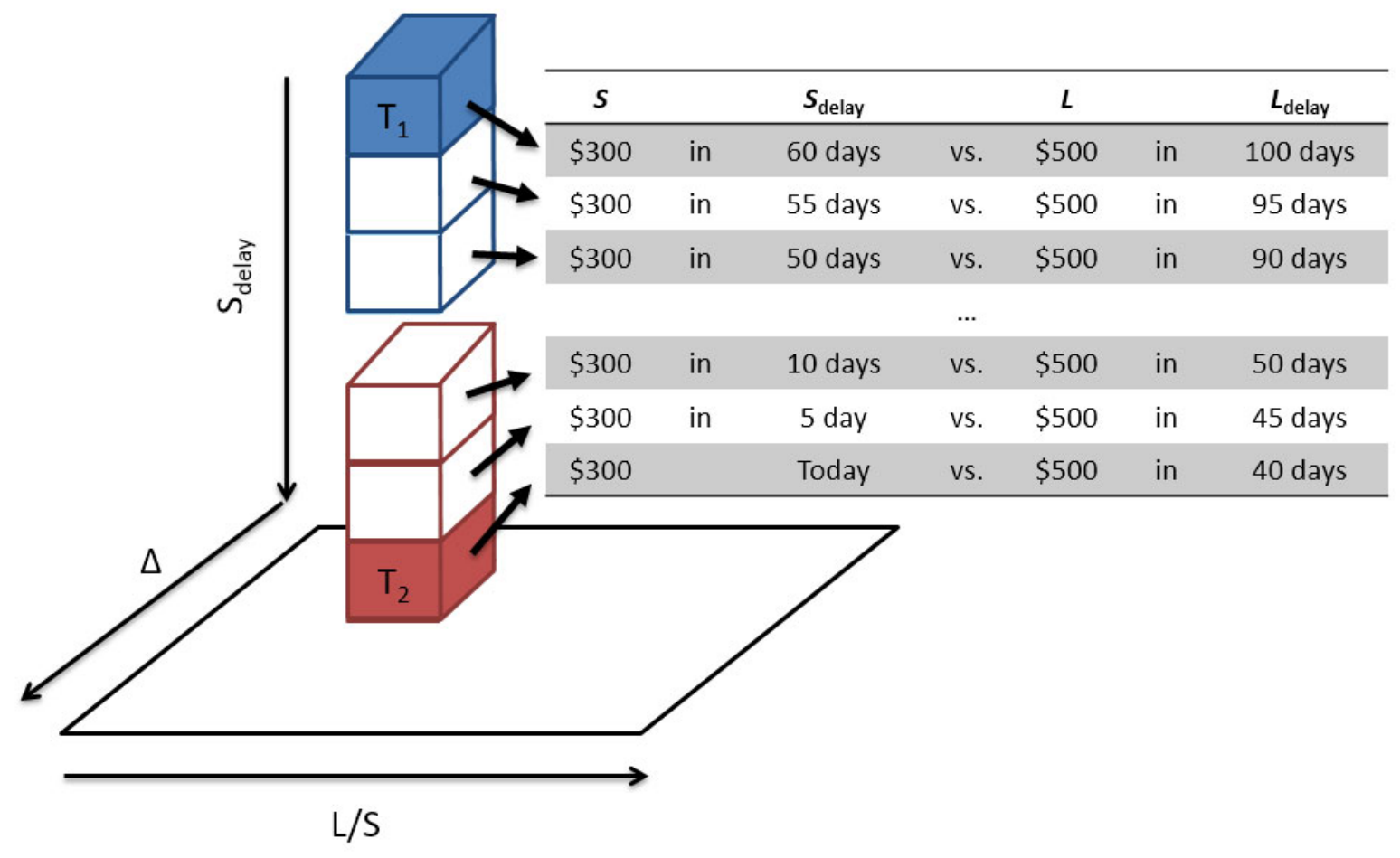

Figure 1. Parameter Space for the List of Intertemporal Choice Questions

Notes. In this example, I held constant $L(\$ 500)$ and the maximum delay for the larger, later option (MLD, 100 days). These variables are expanded in Appendix B. The L/S array (x-axis) was constructed by varying $S$ from $\$ 1$ to $\$ 499$. The $S_{\text {delay }}$ and $\Delta$ arrays range from 0 to 99 days and from 1 to 100 days, respectively, such that they do not exceed the MLD. The stacked boxes represent a series of intertemporal choice questions with $S=\$ 300, L=\$ 500, \Delta=40$ days, and $S_{\text {delay }}$ ranging from 0 to 60 days. 


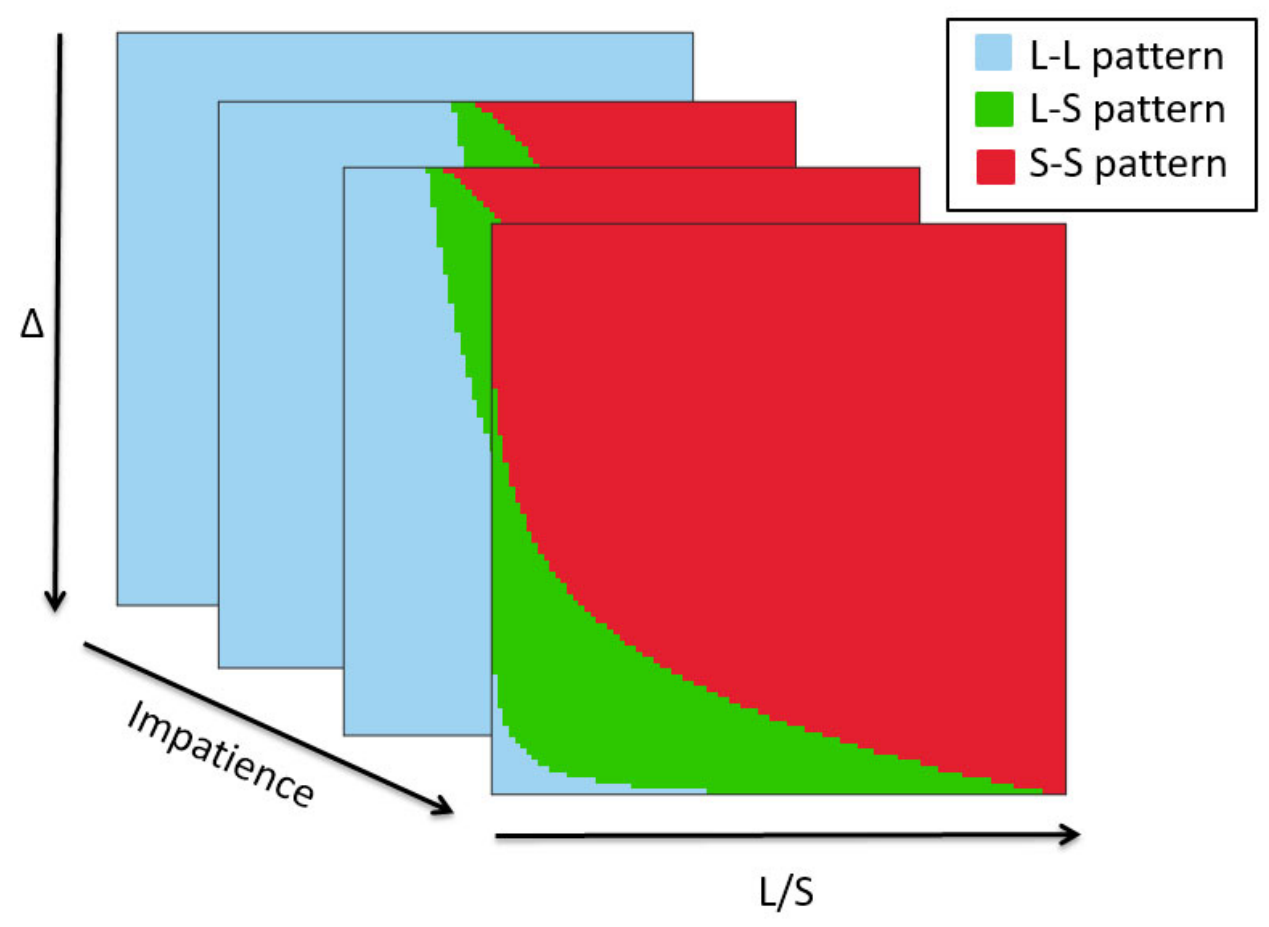

Figure 2. Categorized Choice Patterns

Notes. The parameter space in Figure 1 can be collapsed across the $S_{\text {delay }}$ dimension, creating the twodimensional slices (defined by the $\Delta$ and $L / S$ parameters) shown in this figure. Blue, green, and red spaces designate the parameter combinations that yield the L-L, L-S, and S-S patterns, respectively. 
(a) Exponential

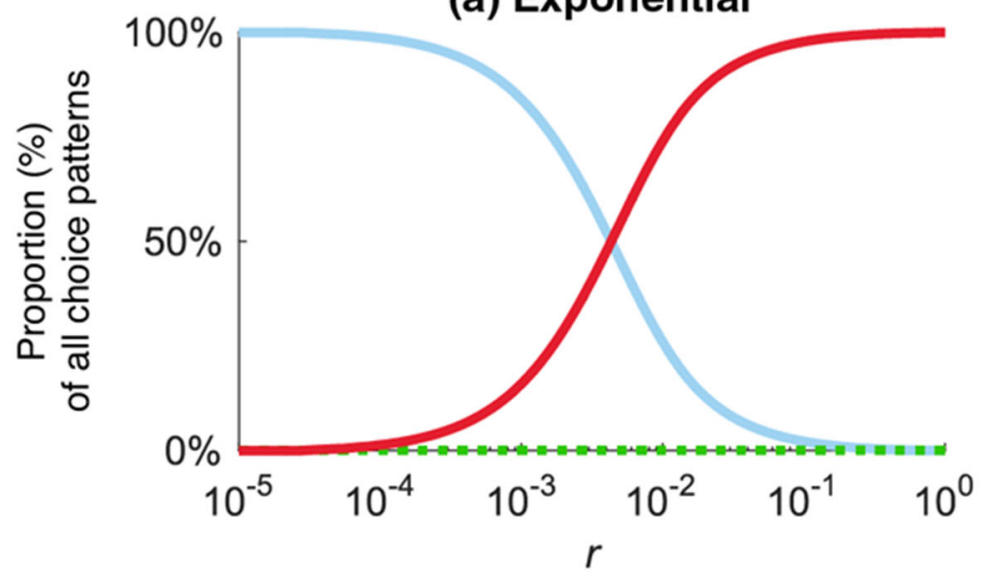

(c) Quasi-hyperbolic $(\beta=.7)$

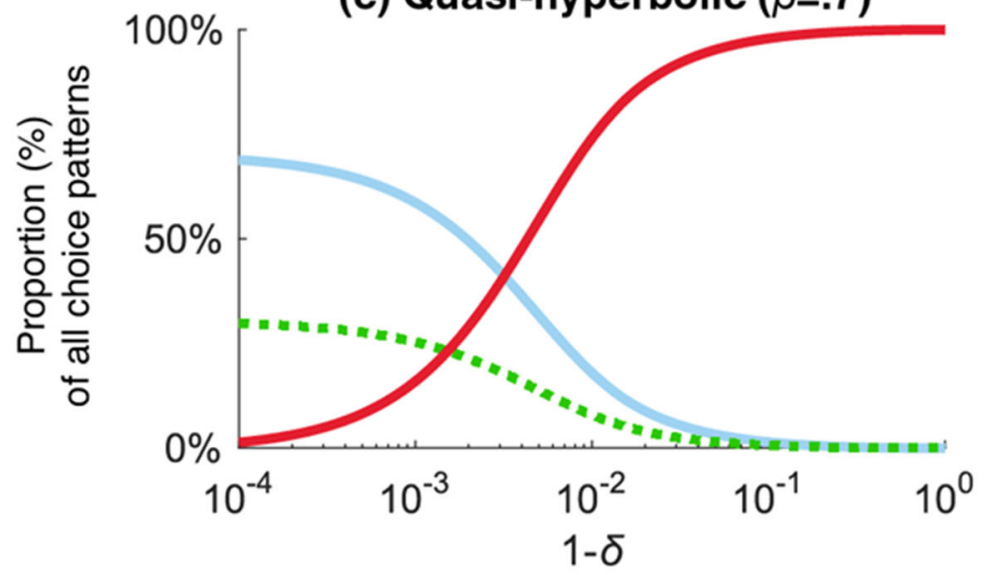

Impatience (b) Hyperbolic

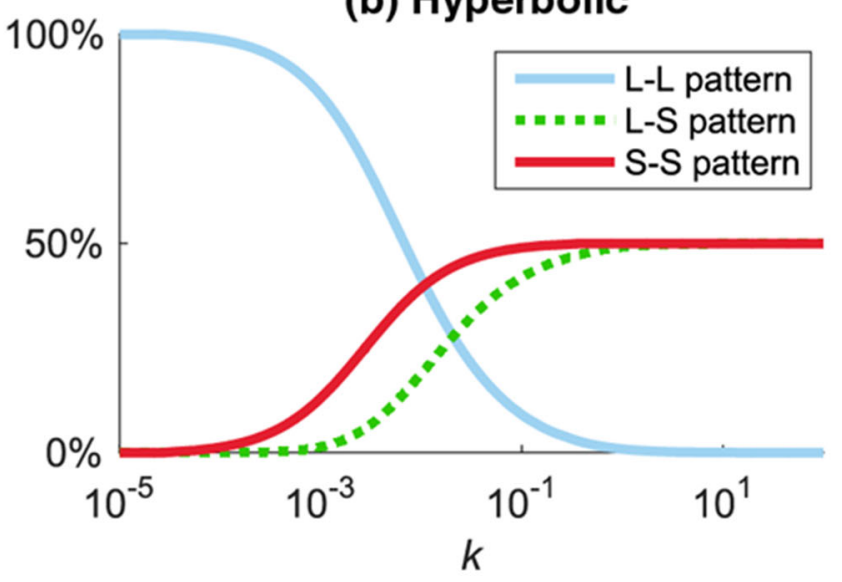

(d) Constant-sensitivity $(b=.5)$

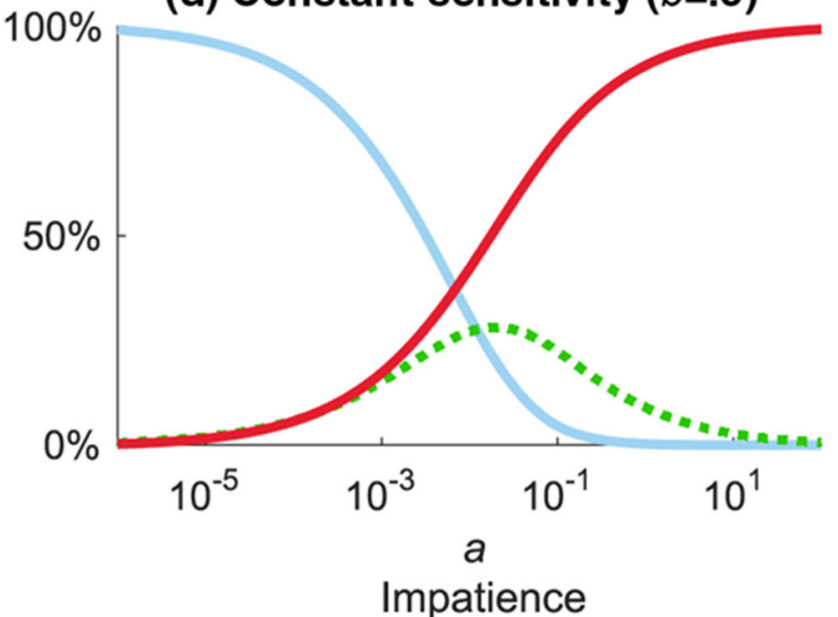

Figure 3. Model Predictions

Notes. The y-axis represents the proportions of the three choice patterns (L-L, L-S, and S-S) that occur at each level of impatience (x-axis). Figure A4 shows additional model predictions for the quasi-hyperbolic and constant-sensitivity models with variations in $\beta$ and $b$. Note that these model predictions are quite different from predictions using the curve fitting technique (Appendix C). 


\subsection{Results}

\section{Exponential model}

The exponential model demonstrated time-consistent choice patterns (Figure 3-a). As expected, the analysis did not show the L-S pattern at any level of impatience because the model was not intended to demonstrate time-inconsistent preferences (Koopmans, 1960). The analysis revealed a gradual transition from $100 \% \mathrm{~L}-\mathrm{L}$ to $100 \% \mathrm{~S}-\mathrm{S}$ as impatience (operationalized as $r$ ) increased. In other words, the model predicted a greater proportion of the L-L pattern with low impatience (smaller $r$ ) and a greater proportion of the S-S pattern with high impatience (larger $r$ ). This gradual transition from the L-L pattern to the S-S pattern with increasing impatience served as a benchmark for subsequent discounting models that did incorporate L-S as a time-inconsistent choice pattern.

\section{Hyperbolic model}

The hyperbolic model demonstrated an asymmetric distribution of the L-S pattern across different levels of impatience (Figure 3-b). As with the exponential model, the hyperbolic model demonstrated a gradual decrease in the proportion of the L-L pattern from $100 \%$ to $0 \%$ as impatience (operationalized as $k$ ) increased. The proportion of the S-S pattern increased as impatience increased, but only up to $50 \%$, unlike in the exponential model. The L-S and S-S patterns followed a similar trend-greater impatience led to a greater proportion of time-inconsistent preferences, such that both choice patterns reached an asymptote at a proportion of $50 \%$. This suggests that the hyperbolic model makes a unique prediction about the relationship between time inconsistency and impatience; those who are impatient also are more likely to demonstrate time-inconsistent preferences.

\section{Quasi-hyperbolic model}

The quasi-hyperbolic model also demonstrated an asymmetric distribution of the L-S pattern across different levels of impatience, but in the opposite direction to the asymmetry in the hyperbolic model (Figure 3-c). Note that on the x-axis, impatience was operationalized as $1-\delta$, instead of $\delta$, to align with the trends in the other models (i.e., that higher values of the impatience parameter indicate higher impatience). Figure 3-c shows the prediction when $\beta=0.7$. The additional figures in Appendix $\mathrm{D}$ show predictions using different values of $\beta$, indicating that a smaller $\beta$ led to a greater proportion of the L-S pattern (Figure A4, left panels). As with the exponential model, the quasi-hyperbolic model demonstrated a gradual increase in the proportion of the S-S pattern from $0 \%$ to $100 \%$ as impatience increased (i.e., as 1- $\delta$ increased). However, unlike in the exponential model, the proportion of the L-L pattern did not reach $100 \%$ even when patience increased (i.e., when $1-\delta$ became very small). The maximum proportion of the L-L pattern was influenced by $\beta$ (in Figure 3-c, the maximum L-L proportion was capped at $70 \%$ because $\beta=.7$ ), and the L-S pattern comprised the remaining predictions (in Figure 3-c, the maximum L-S proportion was $30 \%$ when $\beta=.7$ ) and gradually became less prevalent as impatience increased. This 
suggests that the quasi-hyperbolic model also makes a unique prediction about the relationship between time inconsistency and impatience; those who are less impatient are more likely to demonstrate timeinconsistent preferences.

\section{Constant-sensitivity model}

The constant-sensitivity model demonstrated a symmetric distribution of the L-S pattern across different levels of impatience. Figure 3-d shows the predictions of the constant-sensitivity model (with $b$ $=0.5$ ). The additional figures in Appendix D describe the predictions using different values of $b$, showing that smaller $b$ led to a greater proportion of the L-S pattern (Figure A4, right panels). Unlike in the hyperbolic or quasi-hyperbolic models, the proportions of the S-S and L-L patterns in the constantsensitivity model closely followed those of the exponential model; as impatience (operationalized as $a$ ) increased, there was a gradual shift from $100 \%$ L-L to $100 \%$ S-S. The constant-sensitivity model demonstrated a greater proportion of the L-S pattern during this transition from predominantly L-L to predominantly S-S; the proportion of the L-S pattern peaked with a moderate level of impatience. This suggests that the constant-sensitivity model also makes a unique prediction about the relationship between time inconsistency and impatience; those with moderate impatience are more likely to demonstrate timeinconsistent preferences.

\section{Experiment}

This experiment compared behavioral responses with the model predictions for each of the discounting models. For each participant, I measured the frequency of each of the three choice patterns and elicited the participant's discount rate as a proxy for impatience. Then, I plotted the observed proportion of each choice pattern as a function of impatience, and I compared this plot with the model prediction plots. In this way, I examined the empirical relationship between impatience and time inconsistency, and I compared the model fit of each of the discounting models with regard to that empirical relationship.

\subsection{Method}

\section{Participants}

One hundred participants were recruited online from Amazon Mechanical Turk (40 women; mean age $=31.7$ years). Eighty participants were college graduates, and 11 of those completed postgraduate education as well. The median income range was $\$ 35,000-\$ 49,999$. Participation was restricted to US residents aged 18 or older. 


\section{Attention-check questions}

Sixteen attention-check questions were distributed randomly throughout the main set of questions in the form of choice pairs with an obvious answer (e.g., "Would you prefer \$7.69 in 196 days or \$10.00 today?"). The attention-check questions used the same ranges of amounts and delays as the main questions, so participants needed to pay attention to avoid making mistakes. Before the experiment, I set an exclusion criterion for participants who made more than two mistakes, but none did.

\section{Procedure}

The experimental stimuli were 304 intertemporal choice questions (288 main questions and 16 attention-check questions) presented in a random order. The 288 main questions formed the top and bottom layers of the parameter space (see Figure 1, top and bottom cells). The bottom layer (144 questions) consisted of choices between a smaller, immediate option and a larger, delayed option, and the top layer (144 questions) consisted of questions in which both options were delayed. The 144 questions in each layer were formed by crossing 12 values of $\Delta$ (ranging from 28 to 336 days, with MLD of 364 days) with 12 values of $S$ (ranging from $\$ 0.77$ to $\$ 9.23$, with $L$ fixed at $\$ 10$ ). Choice patterns were identified by comparing the responses to questions from the top layer with the corresponding responses to questions from the bottom layer.

\section{Payment}

Participants received a $\$ 1.50$ base payment upon entering the experiment and a $\$ 1.00$ bonus if they correctly answered at least 14 of the 16 attention-check questions. In addition, all participants received payment in accordance with the outcome of one of their choices, randomly selected. These payments ranged from $\$ 0.77$ to $\$ 10$, with delays ranging from 0 days (i.e., the day of the experiment) to 364 days, and they were paid in the form of Amazon digital gift cards that were delivered on the appropriate date. The average payment was $\$ 8.36$ with a delay of 162 days. In total, participants received an average of $\$ 10.86$ for the 30-minute experiment.

\subsection{Results}

The 288 intertemporal choices yielded 144 choice patterns for each participant. Overall, participant responses exhibited the following distribution of patterns: $46.24 \% \mathrm{~L}-\mathrm{L}, 33.03 \% \mathrm{~S}-\mathrm{S}, 18.62 \%$ L-S, and 2.11\% S-L. ${ }^{4}$ Of the 100 participants, 96 had at least one response with the L-S pattern. Among

\footnotetext{
${ }^{4}$ Overall, the current experiment demonstrated time-consistent preferences (L-L and S-S patterns) in $79.3 \%$ of the choices. This is comparable to previous studies that challenged hyperbolic discounting based on predominant timeconsistent responses from participants (e.g., Andersen et al. 2014, Andreoni and Sprenger 2012, Halevy 2015, Read, Frederick, and Airoldi 2012). Hyperbolic discounting models, however, also predict time-consistent preferences. See Appendix A.
} 
the four participants who did not, one participant always responded with the S-S pattern, one always responded with the L-L pattern, one always responded with the L-L pattern except for one response with the S-L pattern, and one responded with a mixture of the L-L pattern (132 observations) and the S-S pattern (12 observations).

Next, I estimated each participant's discount rate as a proxy for impatience. I used the compound interest rate formula, $\mathrm{PV}=\mathrm{FV} /(1+\mathrm{r})^{\mathrm{n}}$, and for each choice pair, I solved the formula to calculate the discount rate $(r)$ that would make the two options equivalent:

$$
r=e^{\frac{\log (S)-\log (L)}{S_{\text {delay }}-L_{\text {delay }}}}-1
$$

To estimate the discount rate for each participant, I used the elicitation technique from Kirby and Maraković (1996). The scatter plots in Figure 4 show the observed proportions of the three choice patterns as a function of estimated impatience. Locally weighted scatterplot smoothing (LOESS) regression curves $(\alpha=0.7$ and $\lambda=2)$ were superimposed on the three scatter plots. Figure 5 compares the simulation predictions for the constant-sensitivity model (Figure 5-a) with the three LOESS regression curves (Figure 5-b).
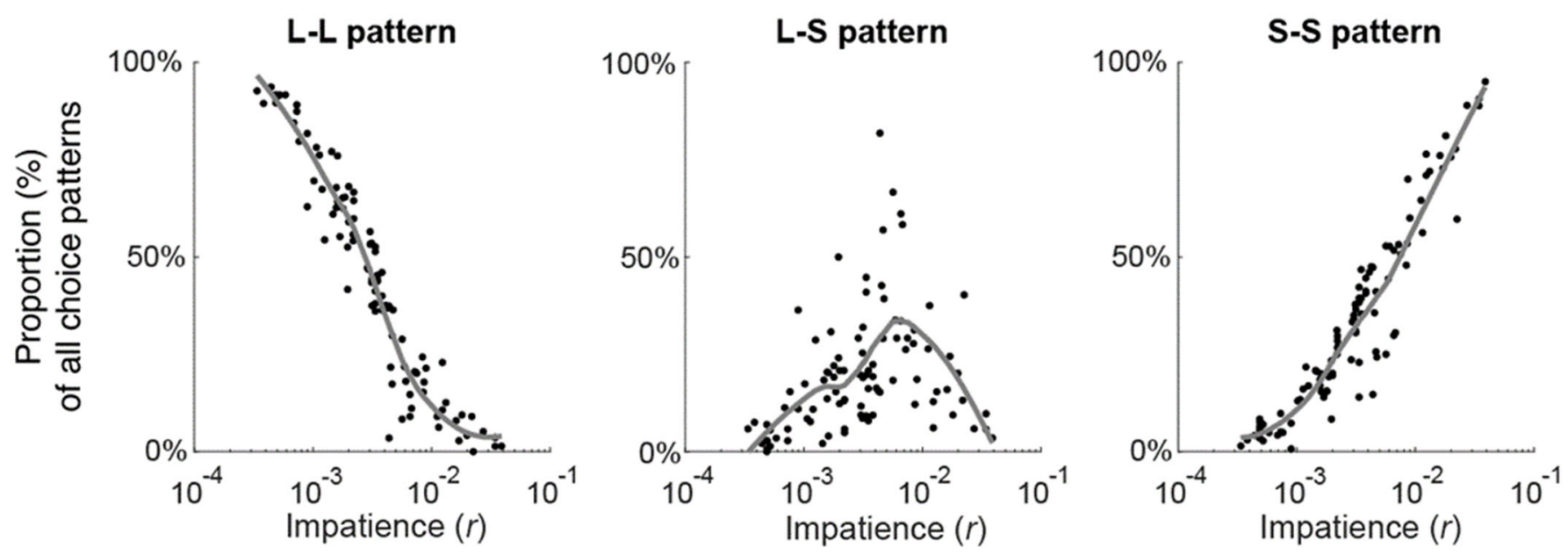

Figure 4. Proportion of Observed Choice Patterns as a Function of Participants' Impatience

Notes. Lines represent LOESS regression curves. 

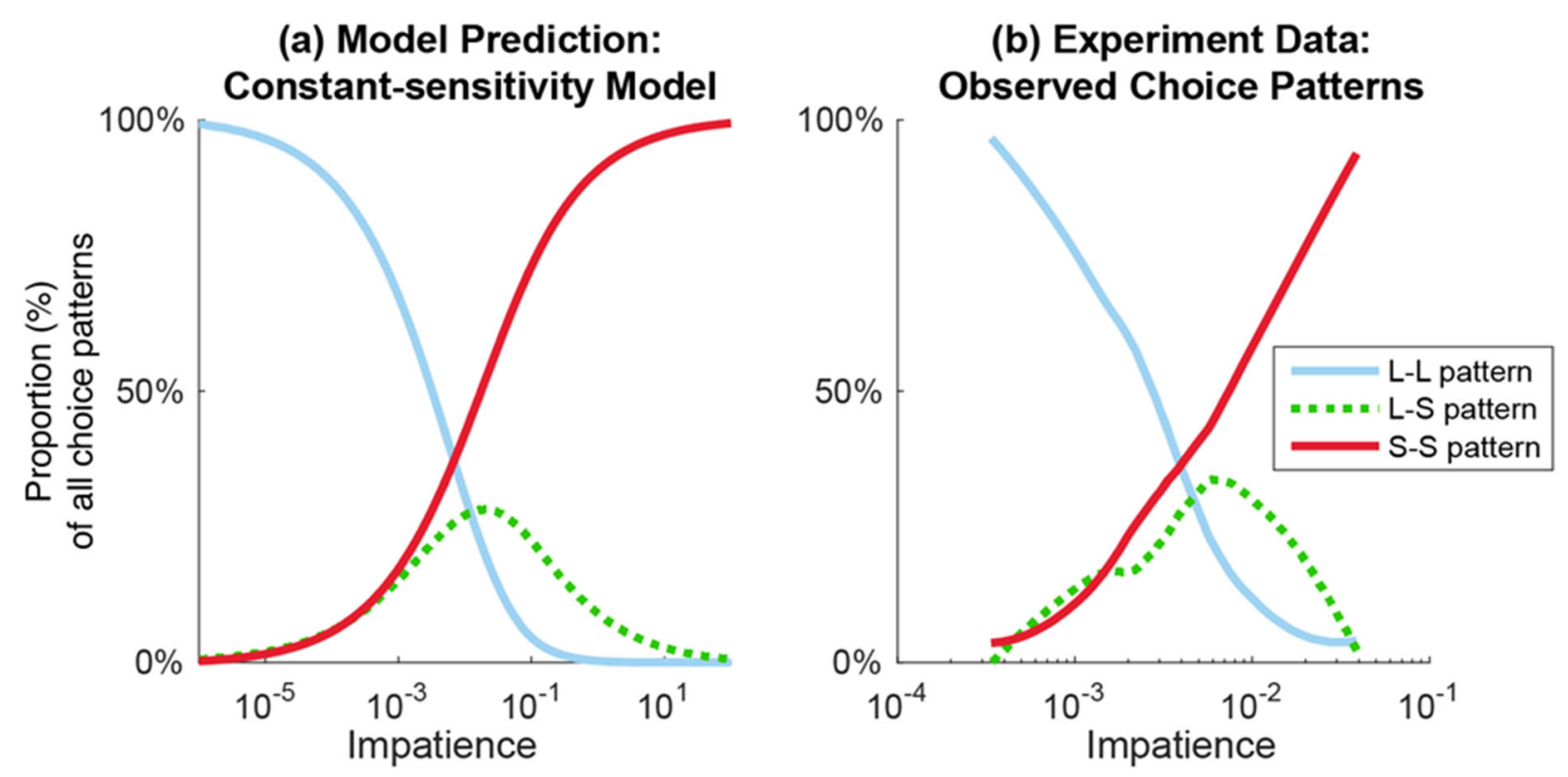

Figure 5. Model Predictions from the Constant-Sensitivity Model $(b=0.5)$ Juxtaposed with the Observed Choice Patterns Using LOESS Regression Curves

Lastly, I calculated the model fit using the maximum likelihood estimation. First, the model simulation provided the predicted proportion of each choice pattern $\left(p S S_{m}, p L S_{m}\right.$, and $\left.p L L_{m}\right)$ for each parameter value $(m)$. Second, from the empirical data, I determined the actual number of observations of each choice pattern $\left(c S S_{i}, c L S_{i}\right.$, and $\left.c L L_{i}\right)$ from each participant $(i)$. Given these two quantities, I calculated the likelihood of each parameter value for each participant, $L(m \mid i)$, using a multinomial distribution:

$$
L(m \mid i)=\frac{c S S_{i}+c L S_{i}+c L L_{i}}{c S S_{i} ! c L S_{i} ! c L L !} p S S_{m} p L S_{m} p L L_{m}
$$

For each participant, I determined the parameter value with the maximum likelihood and calculated the sum of the maximum log-likelihood $(L g L k)$ for each model:

$$
L g L k=-\sum \log _{10} L(m \mid i)_{\max }
$$


The sums of the maximum log-likelihoods provide summary statistics for the model fits across the discounting models. ${ }^{5}$ Because of the negative sign, a lower score implies a better fit. The loglikelihood (base 10) was $1075(b=0.5)$ for the constant-sensitivity model, 1607 for the hyperbolic model, and 2028 ( $\beta=0.7)$ for the quasi-hyperbolic model, meaning that model fit for the constant-sensitivity model was $10^{532}$ times better than that of the hyperbolic model, and $10^{953}$ times better than that of the quasi-hyperbolic model. The sum of the log-likelihoods for the exponential model was infinite, suggesting that this model could not explain the observed choice patterns. In short, the empirical results largely favored the constant-sensitivity model over the other discounting models.

\section{Conclusion}

In the literature on intertemporal choice, behavioral models often entangle time inconsistency and impatience. However, prior to the present study, the true relationship between time inconsistency and impatience was largely unknown. Thus, the current study used a new analysis framework to disentangle these two aspects of temporal discounting. I found that each discounting model makes a unique prediction about the relationship between impatience and time inconsistency: the hyperbolic model predicts that impatient people are more likely to show time inconsistency, while the quasi-hyperbolic model predicts that impatient people are less likely to show time inconsistency, and the constant-sensitivity model predicts that moderately impatient people are more likely to show time inconsistency. The experiment demonstrated that highly patient people show the L-L pattern predominantly, while highly impatient people show the S-S pattern predominantly. The L-S pattern occurred between these two extremes; it peaked at moderate levels of impatience and occurred less often among both highly patient and highly impatient people. In other words, with regard to the gym example at the beginning of the paper, people with moderate impatience are the most likely to sign up for a gym membership and yet choose to stay in bed every morning rather than exercise - thus demonstrating time inconsistency. The current study suggests that the constant-sensitivity model most closely demonstrates the relationship between impatience and time inconsistency.

\footnotetext{
${ }^{5}$ The Bayesian information criteria results were $2152(b=0.5)$ for the constant-sensitivity model, 3216 for the hyperbolic model, $4058(\beta=0.7)$ for the quasi-hyperbolic model, and infinite for the exponential model. To avoid overfitting issues with an additional parameter in the quasi-hyperbolic and constant-sensitivity models, the loglikelihood for each was calculated using the best-fitting additional parameters ( $\beta$ and $b$, respectively; see Appendix E).
} 


\section{Funding}

This material is based upon work supported by the National Science Foundation under Grant No. 1156072.

\section{Acknowledgments}

I am grateful to Gretchen Chapman, Carey Morewedge, Linda Salisbury, Randy Gallistel, and the reviewer team at Management Science for valuable comments and thoughtful guidance. 


\section{References}

Ainslie, G. 1975. Specious reward: a behavioral theory of impulsiveness and impulse control. Psychol. Bull. 82(4) 463-496.

Andersen, S., G. W. Harrison, M. I. Lau, E. E. Rutström. 2006. Elicitation using multiple price list formats. Experimental Economics. 9(4) 383-405.

Andersen, S., G. W. Harrison, M. I. Lau, E. E. Rutström. 2014. Discounting behavior: A reconsideration. Europ. Econ. Rev. 71 15-33.

Andreoni, J., C. Sprenger. 2012. Estimating Time Preferences from Convex Budgets. Amer. Econ. Rev. 102 (7) 3333-3356.

DellaVigna, S., U. Malmendier. 2006. Paying not to go to the gym. Amer. Econ. Rev. 96(3) 694-719.

Ebert, J. E. J., D. Prelec. 2007. The fragility of time: Time-insensitivity and valuation of the near and far future. Manage. Sci. 53(9) 1423-1438.

Halevy, Y. 2015. Time Consistency: Stationarity and Time Invariance. Econometrica. 83(1) 335-352.

Herrnstein, R. J. 1990. Rational choice theory: Necessary but not sufficient. Am. Psychol. 45(3) 356-367.

Hoch, S. J., G. F. Loewenstein. 1991. Time inconsistent Preferences and Consumer Self-Control. Journal of Consumer Research. 17(4) 492-507.

Kirby, K. N., N. N. Maraković. 1996. Delay-discounting probabilistic rewards: Rates decrease as amounts increase. Psychonomic Bulletin and Review. 3(1) 100-104.

Koopmans, T. C. 1960. Stationary Ordinal Utility and Impatience. Econometrica. 28(2) 287-309.

Laibson, D. 1997. Golden eggs and hyperbolic discounting. Quart. J. Econ. 112(2) 442-477.

Mazur, J. E. 1987. An adjusting procedure for studying delayed reinforcement. In Michael L. Commons J. E. M., John A. Nevin and Howard Rachlin. Ed., Quantitative Analyses of Behavior: The Effect of Delay and of Intervening Events on Reinforcement Value (Vol. 5). Hillsdale, NJ. Erlbaum. 5573.

O'Donoghue, T., M. Rabin. 1999. Doing it now or later. Amer. Econ. Rev. 89(1) 103-124.

Prelec, D. 2004. Decreasing impatience: A criterion for non-stationary time preference and "Hyperbolic" discounting. Scandinavian Journal of Economics. 106(3) 511-532.

Read, D., S. Frederick, M. Airoldi. 2012. Four days later in Cincinnati: Longitudinal tests of hyperbolic discounting. Acta Psychologica. 140(2) 177-185.

Samuelson, P. A. 1937. A note on measurement of utility. Review of Economic Studies. 4(2) 155-161.

Sayman, S., A. Oncüler. 2009. An investigation of time inconsistency. Manage. Sci. 55(3) 470-482.

Thaler, R. 1981. Some empirical evidence on dynamic inconsistency. Econ. Letters. 8(3) 201-207. 
Tversky, A., P. Slovic, D. Kahneman. 1990. The Causes of Preference Reversal. The American Economic Review. 80(1) 204-217.

Zauberman, G., B. K. Kim, S. A. Malkoc, J. R. Bettman. 2009. Discounting Time and Time Discounting: Subjective Time Perception and Intertemporal Preferences. J. Marketing Res. 46(4) 543-556. 


\section{Appendix A}

\section{Can time-consistent patterns reject hyperbolic discounting?}

Some studies provide evidence that challenges hyperbolic discounting (e.g., Andersen et al. 2014; Andreoni and Sprenger 2012; Halevy 2015; Read, Frederick, and Airoldi 2012). The consensus among these studies is that time inconsistency (as in the L-S pattern), the main phenomenon behind hyperbolic discounting, is observed much less often than time consistency (as in the L-L and S-S patterns). These studies elicited median present-bias parameter values $(\beta)$ close to 1 in the quasi-hyperbolic model, implying dominant time-consistent preferences (Andersen, Harrison, Lau, and Rutström 2014; Andreoni and Sprenger 2012). Halevy (2015) and Read, Frederick, and Airoldi (2012) reported large proportions of time-consistent preferences using a longitudinal study design that asked the same set of questions repeatedly over time to see if individuals would actually switch their preferences on later days. Read, Frederick, and Airoldi (2012) pointed out that most behavioral studies in support of hyperbolic discounting did not test time inconsistency properly - they used a cross-sectional design that asked multiple questions on the same day, instead of using a longitudinal study design.

However, the empirical results from these longitudinal studies are comparable to those of the current cross-sectional study, which demonstrated 79.3\% time-consistent preferences (L-L and S-S) and 18.6\% time-inconsistent preferences (L-S). In a longitudinal study, Read, Frederick, and Airoldi (2012, p. 181) found that around $78 \%$ of preferences were time-consistent, and $11 \%$ were time-inconsistent. Halevy (2015, p. 345) reported time inconsistency in 34-37\% of the preferences in a similar longitudinal design (dynamic present bias in the robustness condition). Andersen, Harrison, Lau, and Rutström (2014, Figure 2) and Andreoni and Sprenger (2012, Figure 3) also reported a notable prevalence of time-inconsistent preferences. Even when the median $\beta$ was close to 1, these studies reported a small but significant proportion of participants who deviated from the median, suggesting time-inconsistent preferences.

My study suggests that the predominant time-consistent choice patterns observed in these prior studies (or the lack of a predominant time-inconsistent choice pattern) do not necessarily reject hyperbolic discounting. Hyperbolic models also predict considerable time-consistent choice patterns (L-L and S-S), as demonstrated in the model simulations (Figure 3). For example, a simulated quasi-hyperbolic decision maker with $\beta$ close to zero (inducing the maximum proportion of the L-S pattern) also demonstrates a significant proportion of time-consistent choice patterns (Appendix D, left panels). In this particular case ( $\beta$ close to zero), when a quasi-hyperbolic agent is highly impatient ( $\delta$ close to zero), nearly all the choice patterns will be time-consistent (S-S). 


\section{Reverse preference reversal}

Several studies (Halevy 2015; Read, Frederick, and Airoldi 2012; Sayman and Oncüler 2009), especially those with a longitudinal design, have also reported a significant "reverse preference reversal" (the S-L pattern), in which smaller, sooner outcomes are preferred initially and larger, later outcomes are preferred later. My study (cross-sectional design) found that only $2.11 \%$ of the choices followed the S-L pattern. It is possible that differences in the study designs (longitudinal vs. cross-sectional) led to different observations, but the delay scales used in each study may account for the discrepancy. Sayman and Oncüler (2009) reported that the S-L pattern is more likely when a choice involves options with short delays (mostly less than a week). Delay differences $(\Delta)$ in both Halevy (2015) and Read, Frederick, and Airoldi (2012) were one week. My own study used long delay differences, ranging from 28 days to 336 days.

The important issue is not whether the S-L pattern can reject hyperbolic discounting, but rather how the S-L and L-S patterns can be reconciled if they are not random noise. According to the studies mentioned above, individuals may demonstrate increasing impatience in some time periods (manifesting as the S-L pattern) and decreasing impatience in other time periods (manifesting as the L-S pattern). The discounting models tested in this study cannot demonstrate such behavior. Further developments will be needed on this topic. 


\section{Appendix B}

The value of $L$ does not affect the overall model prediction because the discounting models tested in the current study are scale-invariant. The figures below demonstrate this using the hyperbolic model. Figure A1 shows that varying $L(\$ 100, \$ 1000$, and $\$ 10,000)$ does not influence the hyperbolic model prediction. This is true for the other discounting models as well.

The value of MLD does change the parameter space but does not yield qualitatively different predictions. Figure A2 shows the prediction of the hyperbolic model with varying values of MLD (100 days, 1000 days, and 10000 days). Note that variations in MLD change only the x-axis of the model predictions. Again, this is true for the other discounting models as well.

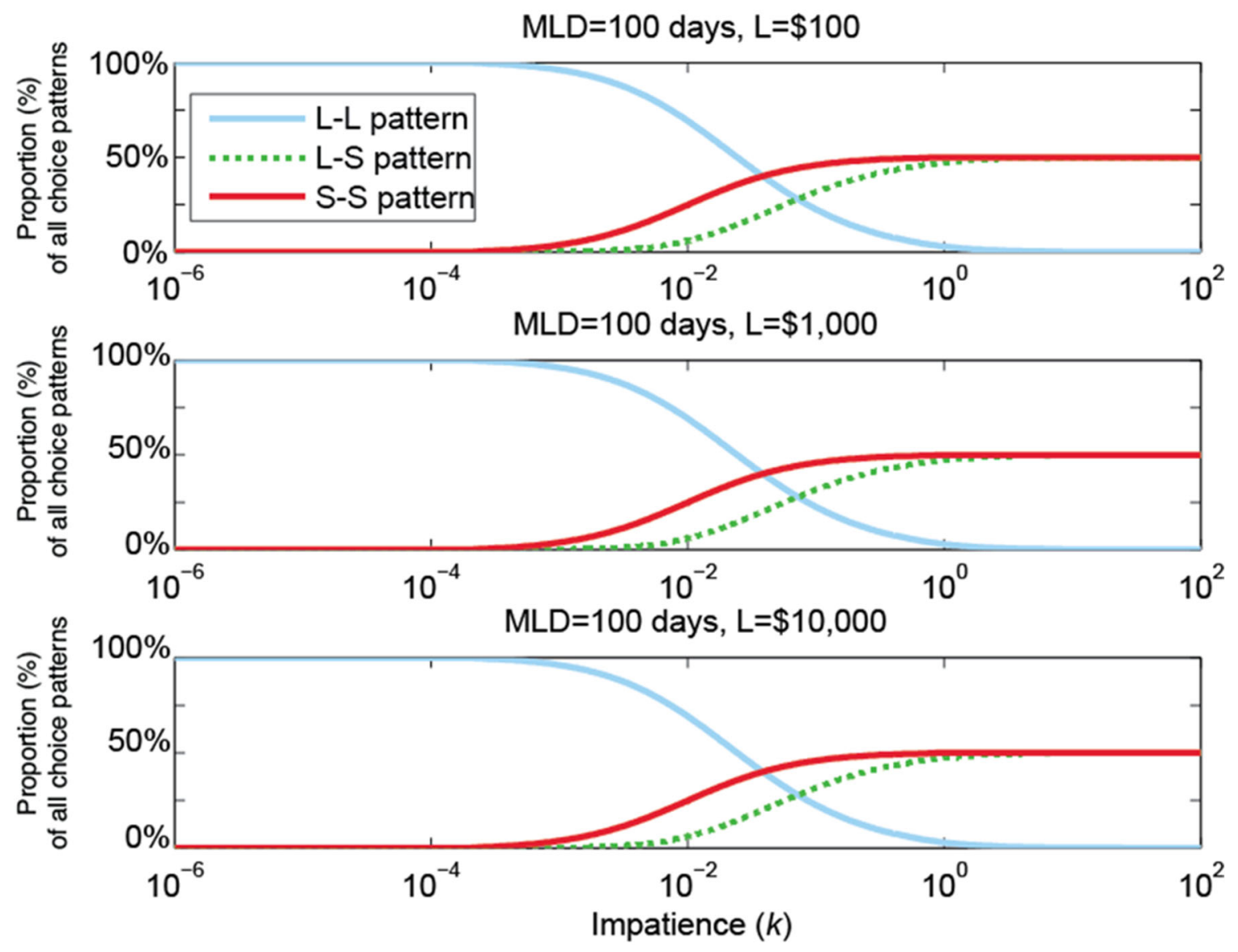

Figure A1: Model Predictions with Varying L Using the Hyperbolic Discounting Model.

Notes. The value of $L$ does not affect the model prediction. 

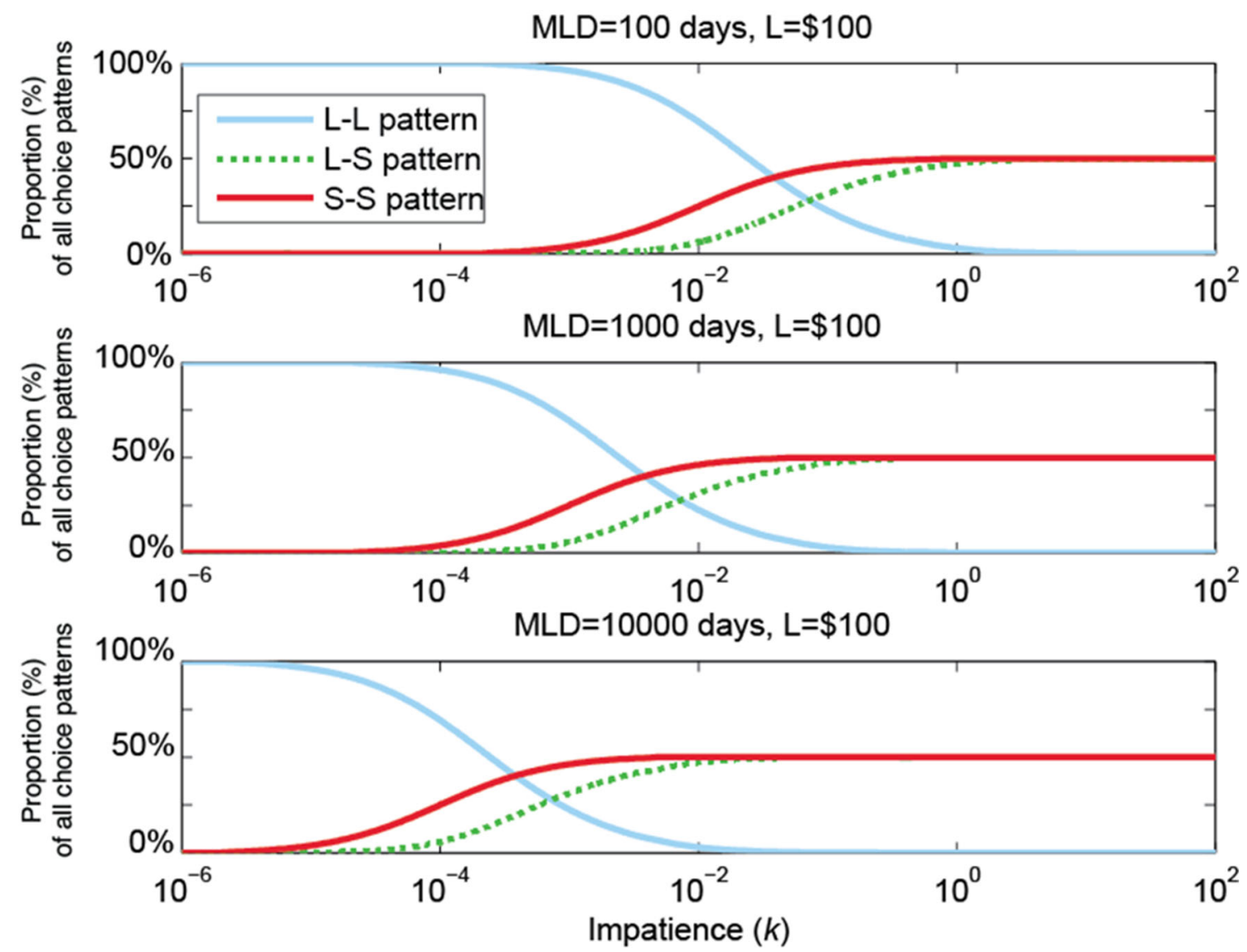

Figure A2: Model Predictions with Varying MLD Using the Hyperbolic Discounting Model.

Notes. As MLD increases, the parameter space shifts to the left. 


\section{Appendix C \\ Curve fitting technique}

This study did not use curve fitting to determine the best model fit. Curve fitting is a common practice for measuring model fit in economics and psychology, as well as in other specific topics such as risk attitude elicitations. In a traditional curve-fitting setting, researchers estimate discounting curves using the list of intertemporal choices (Multiple Price List; for review, see Andersen et al. 2006). Under the assumption that each model has unique curve characteristics, a smaller gap between the empirical and theoretical discounting curves indicates a better model fit.

However, discounting curves from different models are sometimes very similar to each other (Figure A3). For example, the hyperbolic model and the constant-sensitivity model in Figure A3 are almost indistinguishable, even though these models make very different predictions in Figure 3 . There is no denying that curve fitting is a valuable and intuitive technique to explore temporal discounting. Many significant findings have been made using this technique, and it will continue to serve as one of the most convenient tools for researchers. However, it is also equally important to understand its weaknesses, and it should be used with caution when comparing model properties that are not readily distinguishable using the curves.

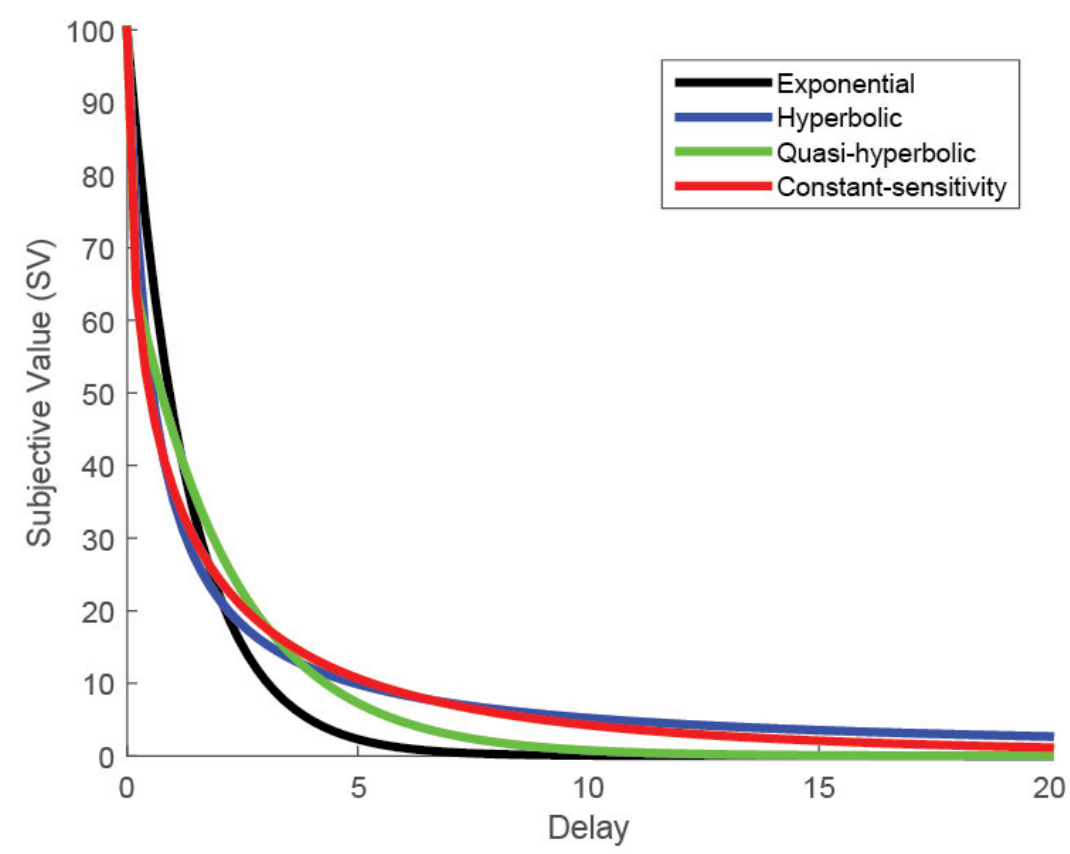

Figure A3. Discounting Curves for the Exponential ( $r=0.75)$, Hyperbolic $(k=1.82)$, QuasiHyperbolic $(\beta=0.7, \delta=0.64)$, and Constant-Sensitivity $(a=1, b=0.5)$ Models 


\section{Appendix D}
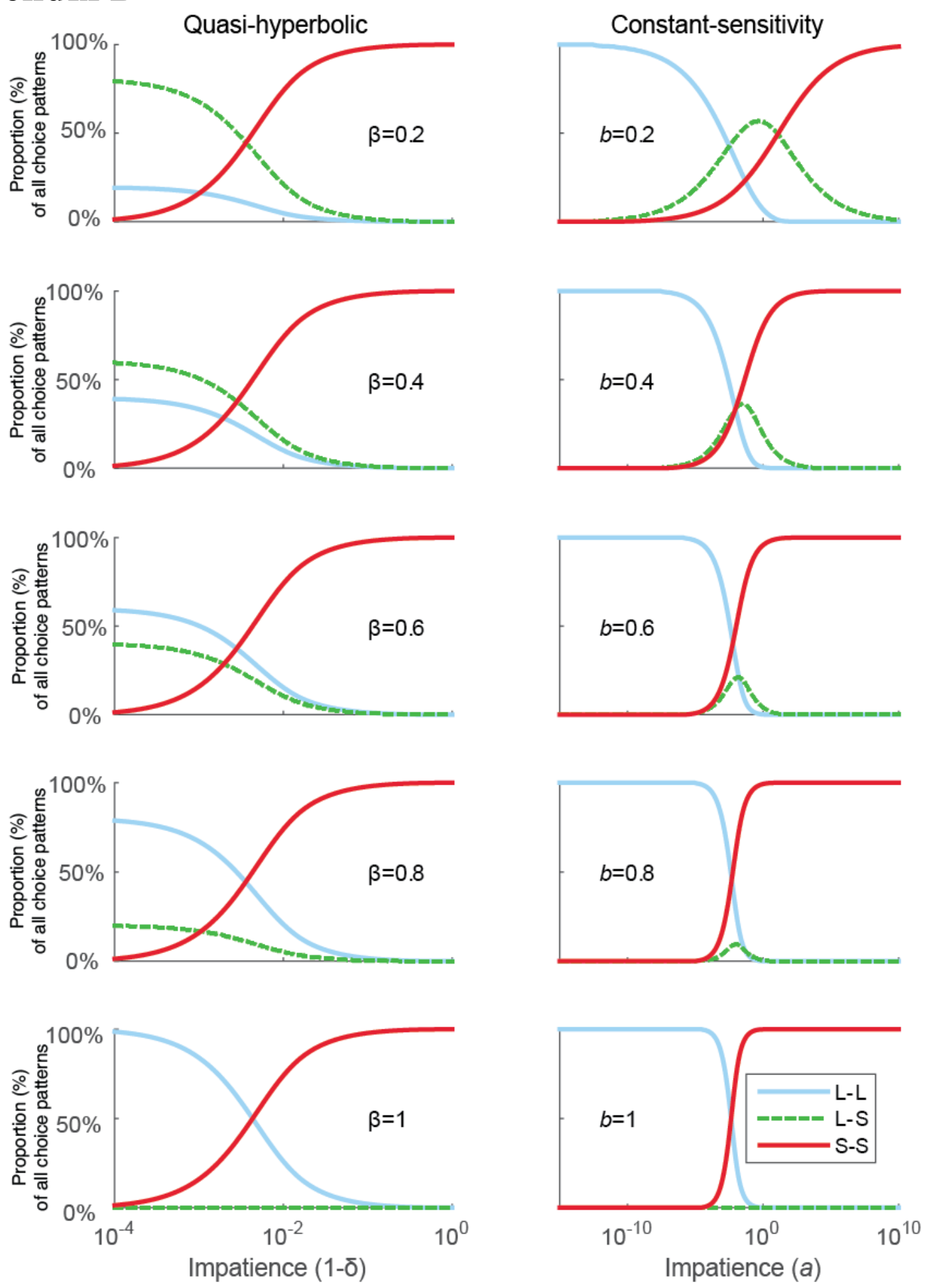

Figure A4: Model Predictions for the Quasi-Hyperbolic Model (Left Column) and the ConstantSensitivity Model (Right Column) with Varying Values of $\beta$ and $b$, Respectively. 


\section{Appendix E \\ Model fitting with different numbers of free parameters}

A model comparison using log-likelihoods should consider different numbers of free parameters.

Both the quasi-hyperbolic and the constant-sensitivity models have two free parameters, while the exponential and hyperbolic models have one free parameter. In general, a model with additional free parameters will fit data better because of the increased flexibility in the model, but it also runs the risk of over-fitting. This was a particular concern because the responses in the current experiment had two degrees of freedom, and the quasi-hyperbolic and constant-sensitivity models had two free parameters to fit the data.

To enable a fair comparison, especially for the exponential and the hyperbolic models, both the quasi-hyperbolic and constant-sensitivity models were converted into a series of single-free-parameter models with variation in the present-bias parameter and time-sensitivity parameter $(\beta$ or $b$ for the two models, respectively). For example, instead of allowing the constant-sensitivity model to have two free parameters, the model was sliced into several single-free-parameter models $\left(f(t)=\exp \left(-(a t)^{0.1}\right), f(t)=\right.$ $\exp \left(-(a t)^{0.2}\right), f(t)=\exp \left(-(a t)^{0.3}\right) \ldots$, where $a$ was the only free parameter as in the exponential and hyperbolic models). The same analysis was also conducted for the quasi-hyperbolic model, treating $\delta$ as a free parameter while varying $\beta$. Finally, the best-fitting parameters $(\beta=0.7$ for the quasi-hyperbolic model and $b=0.5$ for the constant-sensitivity model) were determined by the summed maximum loglikelihood across all the parameter sets (Figure A5). 


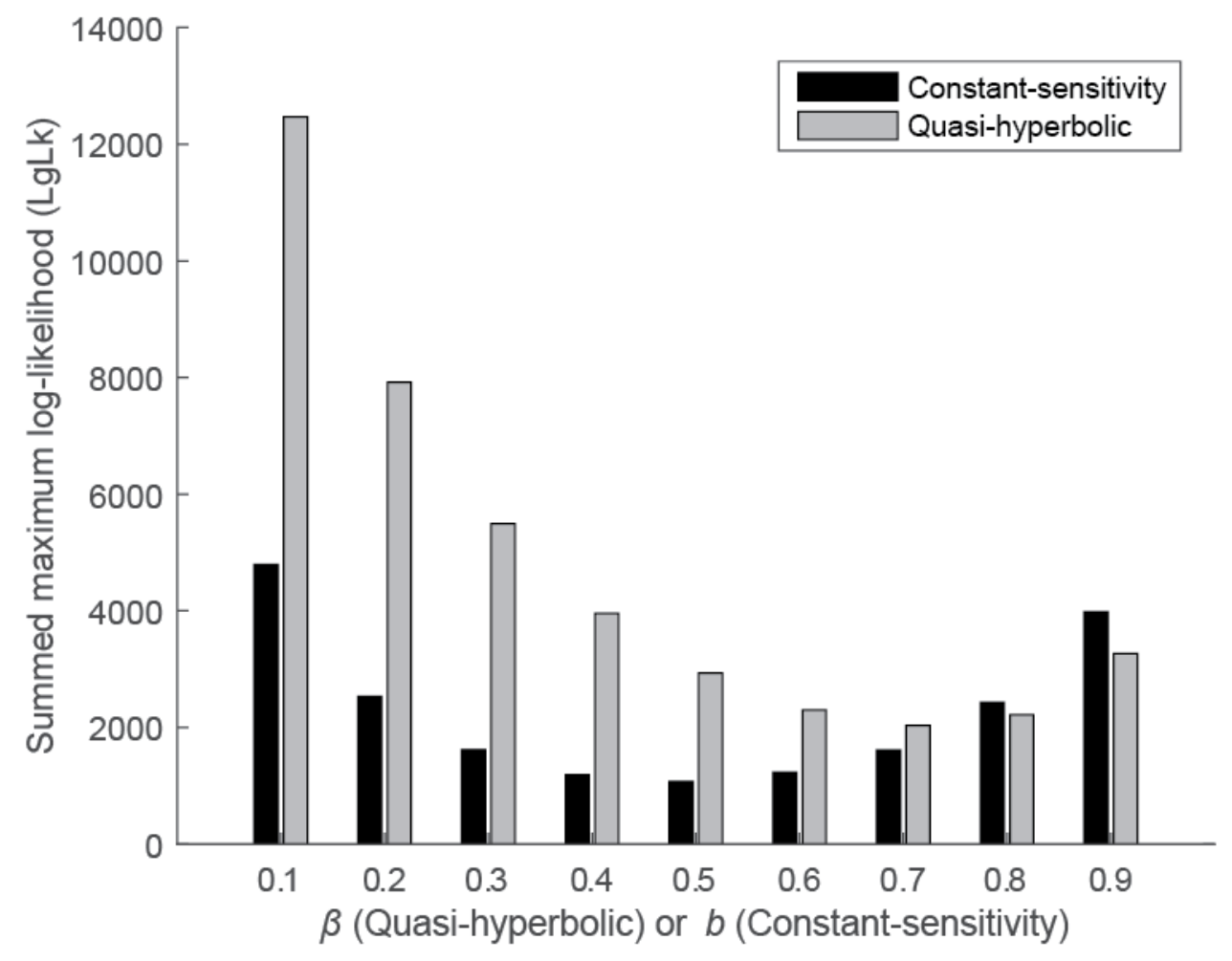

Figure A5. The Sum of the Maximum Log-Likelihood ( $L g L k)$ for the Quasi-Hyperbolic Model and the Constant-Sensitivity Model Across a Range of the $\beta$ and $b$ Parameters

Notes. Because of the negative sign and log scale (base 10) in the equation ( $L g L k)$, smaller numbers imply higher log-likelihoods, meaning better model fit. The best-fitting parameters were $\beta=0.7$ for the quasihyperbolic model and $b=0.5$ for the constant-sensitivity model. 